\title{
HIGHER SYMMETRIES OF THE SQUARE OF THE LAPLACIAN
}

\author{
MICHAEL EASTWOOD AND THOMAS LEISTNER \\ In memory of Thomas Branson
}

\begin{abstract}
The symmetry operators for the Laplacian in flat space were recently described and here we consider the same question for the square of the Laplacian. Again, there is a close connection with conformal geometry. There are three main steps in our construction. The first is to show that the symbol of a symmetry is constrained by an overdetermined partial differential equation. The second is to show existence of symmetries with specified symbol (using a simple version of the AdS/CFT correspondence). The third is to compute the composition of two first order symmetry operators and hence determine the structure of the symmetry algebra. There are some interesting differences as compared to the corresponding results for the Laplacian.
\end{abstract}

\section{INTRODUCTION}

The second order symmetry operators for the Laplacian on $\mathbb{R}^{n}$ were determined by Boyer, Kalnins, and Miller [2]. The higher order symmetries were found in 6] and the structure of the resulting algebra was also described. Here were prove the corresponding results for the square of the Laplacian. The other aspect of [2], namely the relation between second order symmetries and separation of variables, is unclear for the square of the Laplacian.

We are grateful to Ernie Kalnins who suggested the square of the Laplacian as a candidate for having interesting symmetries. We would also like to acknowledge pertinent comments from Petr Somberg, Vladimír Souček, and Misha Vasiliev.

\section{Definitions And Statements of REsults}

We shall always work on $n$-dimensional Euclidean space $\mathbb{R}^{n}$ for $n \geq 3$ and adopt the usual convention of writing vectors and tensors adorned with indices, which we shall raise and lower with the standard (flat) metric $g_{a b}$. Let us also write $\nabla_{a}=\partial / \partial x^{a}$ for differentiation in coördinates. Then $\nabla^{a}=g^{a b} \nabla_{b}$ and the Laplacian is given by $\Delta=\nabla^{a} \nabla_{a}$. All functions and tensors in this article will be smooth. All differential operators will be linear with smooth coefficients.

2000 Mathematics Subject Classification. Primary 58J70; Secondary 16S32, 53A30, 70S10.

Key words and phrases. Symmetry algebra, Laplacian, Conformal geometry.

This work was undertaken in preparation for and during the 2006 Summer Program at the Institute for Mathematics and its Applications at the University of Minnesota. The authors would like to thank the IMA for hospitality during this time. The authors are supported by the Australian Research Council. 
Definition 1. A differential operator $\mathcal{D}$ is a symmetry of $\Delta^{2}$ if and only if there is another differential operator $\delta$ such that $\Delta^{2} \mathcal{D}=\delta \Delta^{2}$.

Obviously, any operator of the form $\mathcal{P} \Delta^{2}$ is a symmetry of $\Delta^{2}$ because we can take $\delta=\Delta^{2} \mathcal{P}$. Therefore one introduces the following equivalence relation.

Definition 2. Two symmetries $\mathcal{D}_{1}$ and $\mathcal{D}_{2}$ of $\Delta^{2}$ are equivalent, $\mathcal{D}_{1} \sim \mathcal{D}_{2}$, if and only if $\mathcal{D}_{1}-\mathcal{D}_{2}=\mathcal{P} \Delta^{2}$ for some differential operator $\mathcal{P}$.

Of course, this equivalence relation only effects symmetries of order $s \geq 4$. The composition of two symmetries is again a symmetry. Also, composition is compatible with the equivalence relation, i.e. if $\mathcal{D}_{1} \sim \mathcal{D}_{2}$ and $\mathcal{D}_{3} \sim \mathcal{D}_{4}$, then $\mathcal{D}_{1} \mathcal{D}_{3} \sim \mathcal{D}_{2} \mathcal{D}_{4}$. This allows us to define an algebra:-

Definition 3. The algebra $\mathcal{B}_{n}$ consists of all symmetries of $\Delta^{2}$ on $\mathbb{R}^{n}$ considered modulo equivalence and with algebra operation induced by composition.

In the following we shall study this algebra and describe its structure. To this end we need the notion of conformal Killing tensors and their generalisations as studied in [10, 11]. We shall write $\phi^{(a b \cdots c)}$ for the symmetric part of a tensor $\phi^{a b \cdots c}$.

Definition 4. A conformal Killing tensor $V^{b c d \cdots e}$ is a symmetric trace-free tensor such that

$$
\text { the trace-free part of } \nabla^{(a} V^{b c d \cdots e)}=0 \text {, }
$$

equivalently that

$$
\nabla^{(a} V^{b c d \cdots e)}=g^{(a b} \phi^{c d \cdots e)}
$$

for some tensor $\phi^{\text {cd } \cdots e}$. A conformal Killing tensor with one index is called a conformal Killing vector. A conformal Killing tensor with no indices is simply a constant.

Definition 5. A generalised conformal Killing tensor $W^{d \cdots e}$ of order 3 is a symmetric trace-free tensor such that

$$
\text { the trace-free part of } \nabla^{(a} \nabla^{b} \nabla^{c} W^{d \cdots e)}=0,
$$

equivalently that

$$
\nabla^{(a} \nabla^{b} \nabla^{c} W^{d \cdots e)}=g^{(a b} \phi^{c d \cdots e)}
$$

for some tensor $\phi^{c d \cdots e}$.

Though it is clear how to define a generalised conformal Killing tensor of any order, we shall only need order 3. This should be taken as read for the rest of this article.

Our main theorems on the existence and uniqueness of symmetries are as follows.

Theorem 1. Any zeroth order symmetry of $\Delta^{2}$ is of the form

$$
f \longmapsto V f \quad \text { for } V \text { constant. }
$$

Any first order symmetry of $\Delta^{2}$ is of the form

$$
V^{b} \nabla_{b}+\text { lower order terms, }
$$


where $V^{b}$ is a conformal Killing vector. Any higher symmetry, say of degree s, of $\Delta^{2}$ is canonically equivalent to one of the form

$$
V^{b c d \cdots e} \nabla_{b} \nabla_{c} \nabla_{d} \cdots \nabla_{e}+W^{d \cdots e} \Delta \nabla_{d} \cdots \nabla_{e}+\text { lower order terms, }
$$

where $V^{\text {bcd } \cdots e}$ is a conformal Killing tensor of valency $s$ and $W^{d \cdots e}$ is a generalised conformal Killing tensor of valency $s-2$.

Theorem 2. Suppose that $V^{b c d \cdots e}$ is a conformal Killing tensor on $\mathbb{R}^{n}$. Then there is a canonically defined differential operator

$$
\mathcal{D}_{V}=V^{b c d \ldots e} \nabla_{b} \nabla_{c} \nabla_{d} \cdots \nabla_{e}+\text { lower order terms }
$$

that is a symmetry of $\Delta^{2}$. Suppose that $W^{d \cdots e}$ is a generalised conformal Killing tensor. Then there is a canonically defined differential operator

$$
\mathcal{D}_{W}=W^{d \ldots e} \Delta \nabla_{d} \cdots \nabla_{e}+\text { lower order terms }
$$

that is a symmetry of $\Delta^{2}$.

The proof of this theorem will be given in Section 4 by using the ambient metric construction. Here we only want to give the first and second order symmetries. As a special case of (14), any first order symmetry is given by

$$
\mathcal{D} f=V^{a} \nabla_{a} f+\frac{n-4}{2 n}\left(\nabla_{a} V^{a}\right) f+c f,
$$

for a conformal Killing vector $V^{a}$ and arbitrary constant $c$. The canonical ones $\mathcal{D}_{V}$ are those with $c=0$. The canonical second order symmetries are

$$
\mathcal{D}_{V} f=V^{a b} \nabla_{a} \nabla_{b} f+\frac{n-2}{n+2}\left(\nabla_{a} V^{a b}\right) \nabla_{b} f+\frac{(n-2)(n-4)}{4(n+1)(n+2)}\left(\nabla_{a} \nabla_{b} V^{a b}\right) f
$$

for $V^{a b}$ a conformal Killing tensor and

$$
\mathcal{D}_{W} f=W \Delta f-\left(\nabla^{a} W\right) \nabla_{a} f-\frac{n-4}{2(n+2)}(\Delta W) f,
$$

for $W$ a generalised conformal Killing scalar, i.e. $\nabla^{a} \nabla^{b} \nabla^{c} W=g^{(a b} \phi^{c)}$. Of course, there is no freedom in equivalence until we consider fourth order operators. Hence, we can use Theorems 1 and 2 to write down all second order symmetries as follows. Suppose that $\mathcal{D}$ is a second order symmetry operator. According to Theorem 1 , it has the form

$$
\mathcal{D}=V^{a b} \nabla_{a} \nabla_{b}+W \Delta+\text { lower order terms }
$$

where $V^{a b}$ is a conformal Killing tensor and $W$ is a generalised conformal Killing scalar. According to Theorem 2, however, there are canonically defined symmetry operators of the same form, which we can subtract to obtain a first order symmetry. Iterating this procedure we conclude that

$$
\mathcal{D}=\mathcal{D}_{V_{2}}+\mathcal{D}_{W}+\mathcal{D}_{V_{1}}+\mathcal{D}_{V_{0}}
$$

where $\mathcal{D}_{V_{s}}$ are the canonically defined differential operators associated to conformal Killing tensors of valency $s$ and $\mathcal{D}_{W}$ is the operator associated to a generalised conformal Killing scalar. As above, we have explicit formulae for these operators and, of course, $\mathcal{D}_{V_{0}} f=V_{0} f$ for constant $V_{0}$. We shall soon see that the space of (generalised) 
conformal Killing tensors is finite-dimensional. (In particular, the space of second order symmetries of $\Delta^{2}$ has dimension $(n+1)(n+2)\left(n^{2}+5 n+12\right) / 12$. $)$

More generally, let $\mathcal{K}_{n, s}$ denote the vector space of conformal Killing tensors on $\mathbb{R}^{n}$ with $s$ indices and, for $s \geq 2$, let $\mathcal{L}_{n, s}$ denote the vector space of generalised conformal Killing tensors on $\mathbb{R}^{n}$ with $s-2$ indices. Reasoning as we just did for second order symmetries, but now taking into account the equivalence necessitated by Theorem 1 in general, we conclude that any symmetry of $\Delta^{2}$ may be canonically thrown into an equivalent one of the form

$$
\mathcal{D}_{V_{s}}+\mathcal{D}_{W_{s}}+\cdots+\mathcal{D}_{V_{2}}+\mathcal{D}_{W_{2}}+\mathcal{D}_{V_{1}}+\mathcal{D}_{V_{0}}, \quad \text { for } V_{s} \in \mathcal{K}_{n, s}, W_{s} \in \mathcal{L}_{n, s} .
$$

Another way of stating this is:-

Corollary 1. There is the following canonical isomorphism of vector spaces.

$$
\mathcal{B}_{n} \simeq \mathcal{K}_{n, 0} \oplus \mathcal{K}_{n, 1} \oplus \bigoplus_{s=2}^{\infty}\left(\mathcal{K}_{n, s} \oplus \mathcal{L}_{n, s}\right)
$$

In order the present the algebra structure on $\mathcal{B}_{n}$ we need more detail on the spaces $\mathcal{K}_{n, s}$ and $\mathcal{L}_{n, s}$. It is well-known and given explicitly in (17), that the space of conformal Killing vectors on $\mathbb{R}^{n}$ is isomorphic as a Lie algebra to $\mathfrak{s o}(n+1,1)$. The spaces $\mathcal{K}_{n, s}$ and $\mathcal{L}_{n, s}$ are irreducible finite-dimensional representations of $\mathfrak{s o}(n+1,1)$. Specifically,



and

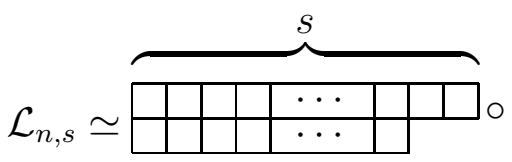

as Young tableau, where $\circ$ denotes the trace-free part. These isomorphisms may be derived from results concerning induced modules in representation theory, namely Lepowsky's generalisation [9] of the Bernstein-Gelfand-Gelfand resolution. A proof in the language of partial differential operators appears in [3].

From now on, let us write $\mathfrak{g}$ for the Lie algebra $\mathfrak{s o}(n+1,1)$. Then

$$
\mathfrak{g} \otimes \mathfrak{g}=\square \otimes \square=\square \square_{\circ} \oplus \square \square \oplus \mathbb{R} \oplus \square_{\square_{0}} \oplus \square \oplus \square
$$

and, following [6], we shall write $X \odot Y$ for the projection

$$
\mathfrak{g} \otimes \mathfrak{g} \ni V \otimes W \longmapsto V \odot W \in \square \square_{\circ}
$$


It is shown in [6] that the symmetry algebra $\mathcal{A}_{n}$ for the Laplacian is isomorphic to the tensor algebra $\otimes \mathfrak{g}$ modulo the two-sided ideal generated by

$$
V \otimes W-V \odot W-\frac{1}{2}[V, W]+\frac{n-2}{4 n(n+1)}\langle V, W\rangle \quad \text { for } V, W \in \mathfrak{g},
$$

where $\langle$,$\rangle is the Killing form (normalised in the usual way, not as in [6]). To state$ the corresponding result for $\mathcal{B}_{n}$ we also need a notation for the projection onto $\square \square$ 。 and we shall write this as $V \otimes W \mapsto V \bullet W$ meaning as an idempotent homomorphism of $\mathfrak{g} \otimes \mathfrak{g}$ into itself. We also need to observe that

$$
\square \square \square \square_{\circ} \hookrightarrow \mathfrak{g} \odot \mathfrak{g} \odot \mathfrak{g} \odot \mathfrak{g} \subset \mathfrak{g} \otimes \mathfrak{g} \otimes \mathfrak{g} \otimes \mathfrak{g}
$$

meaning that there is a unique irreducible summand of the symmetric tensor product $\bigodot^{4} \mathfrak{g}$ of the indicated type. With these conventions in place, we have:-

Theorem 3. The algebra $\mathcal{B}_{n}$ is isomorphic to the tensor algebra $\otimes \mathfrak{g}$ modulo the 2-sided ideal generated by

$$
V \otimes W-V \odot W-V \bullet W-\frac{1}{2}[V, W]+\frac{(n-4)(n+4)}{4 n(n+1)(n+2)}\langle V, W\rangle \quad \text { for } V, W \in \mathfrak{g}
$$

and the image of $\square \square \square$ o in $\otimes^{4} \mathfrak{g}$.

As noted in [6] for $\mathcal{A}_{n}$, we can quotient firstly by $V \wedge W-\frac{1}{2}[V, W]$ to realise $\mathcal{B}_{n}$ as a quotient of $\mathfrak{U}(\mathfrak{g})$, the universal enveloping algebra of $\mathfrak{g}$. Compared to $\mathcal{A}_{n}$, the appearance of additional generators at $4^{\text {th }}$ order is new.

In fact, there is a more precise statement from which Theorem 3 easily follows. It appears as Theorem 4 in Section 5.

\section{The proof of Theorem 1}

Lemma 1. Suppose $V^{b c d \cdots e f}$ is a conformal Killing tensor with s indices. If we define $\phi^{c d \cdots e f}$ according to

$$
\nabla^{(a} V^{b c d \cdots e f)}=g^{(a b} \phi^{c d \cdots e f)}
$$

then

$$
\Delta V^{b c d \cdots e f}=(s-1) g^{(b c} \nabla_{a} \phi^{d \cdots e f) a}-(n+2 s-4) \nabla^{(b} \phi^{c d \cdots e f)}
$$

and

$$
\text { the trace-free part of } \nabla^{(a} \nabla^{b} \phi^{c d \cdots e f)}=0 .
$$

Proof. Taking the trace of (10) gives

$$
\frac{2}{s+1} \nabla_{b} V^{b c d \cdots e f}=\frac{2 n}{s(s+1)} \phi^{c d \cdots e f}+\frac{4(s-1)}{s(s+1)} \phi^{c d \cdots e f}
$$

whence

$$
\phi^{c d \cdots e f}=\frac{s}{n+2 s-2} \nabla_{b} V^{b c d \cdots e f} .
$$

If we apply $\nabla_{a}$ to (10) we obtain

$$
\frac{1}{s+1} \Delta V^{b c d \cdots e f}+\frac{s}{s+1} \nabla^{(b} \nabla_{a} V^{c d \cdots e f) a}=\frac{2}{s+1} \nabla^{(b} \phi^{c d \cdots e f)}+\frac{s-1}{s+1} g^{(b c} \nabla_{a} \phi^{d \cdots e f) a} .
$$


In combination with (12), this completes the proof of (111). Since $n \geq 3$ and $s \geq 1$, the coefficient $n+2 s-4$ is always non-zero and the final conclusion now follows by differentiating (11).

Now, we are in a position to prove Theorem 11. Let us write

$$
\mathcal{D}=T^{a b c d e \cdots f} \nabla_{a} \nabla_{b} \nabla_{c} \nabla_{d} \nabla_{e} \cdots \nabla_{f}+\text { lower order terms, }
$$

where $T^{a b c d e \cdots f}$ is a non-zero symmetric tensor, namely the symbol of $\mathcal{D}$. This tensor splits uniquely as

$$
T^{a b c d e \cdots f}=V^{a b c d e \cdots f}+g^{(a b} W^{c d e \cdots f)}+g^{(a b} g^{c d} X^{e \cdots f)}
$$

where $V^{a b c d e \cdots f}$ and $W^{c d e \cdots f}$ are symmetric trace-free and $X^{e \cdots f}$ is symmetric. By subtracting

$$
\Delta^{2} X^{e \cdots f} \nabla_{e} \cdots \nabla_{f}=X^{e \cdots f} \Delta^{2} \nabla_{e} \cdots \nabla_{f}+\text { lower order terms }
$$

from $\mathcal{D}$ we have found a canonically equivalent symmetry of the form

$$
\mathcal{D}=V^{a b c d e \cdots f} \nabla_{a} \nabla_{b} \nabla_{c} \nabla_{d} \nabla_{e} \cdots \nabla_{f}+W^{c d e \cdots f} \Delta \nabla_{c} \nabla_{d} \nabla_{e} \cdots \nabla_{f}+\text { lower order terms }
$$

and we claim that $V^{\text {abcde } \cdots f}$ must be a conformal Killing tensor and $W^{\text {cde } \cdots f}$ must be a generalised conformal Killing tensor. To see this we simply compute $\Delta^{2} \mathcal{D}$ and for this task it is convenient to use the formula

$$
\begin{gathered}
\Delta^{2}(f g)=f \Delta^{2} g+4\left(\nabla^{a} f\right) \Delta \nabla_{a} g+2(\Delta f) \Delta g+4\left(\nabla^{a} \nabla^{b} f\right) \nabla_{a} \nabla_{b} g \\
+4\left(\Delta \nabla^{a} f\right) \nabla_{a} g+\left(\Delta^{2} f\right) g
\end{gathered}
$$

and its evident extension to tensor expressions. If we write

$$
\begin{aligned}
\mathcal{D}=V^{h i j k l \cdots m} \nabla_{h} \nabla_{i} \nabla_{j} \nabla_{k} \nabla_{l} \cdots \nabla_{m}+W^{j k l \cdots m} \Delta \nabla_{j} \nabla_{k} \nabla_{l} \cdots \nabla_{m} \\
+Y^{i j k l \cdots m} \nabla_{i} \nabla_{j} \nabla_{k} \nabla_{l} \cdots \nabla_{m}+Z^{j k l \cdots m} \nabla_{j} \nabla_{k} \nabla_{l} \cdots \nabla_{m} \\
+ \text { lower order terms }
\end{aligned}
$$

where $Y^{i j k l \cdots m}$ and $Z^{j k l \cdots m}$ are symmetric, then

$$
\begin{aligned}
\Delta^{2} \mathcal{D}=V^{h i j \cdots m} & \Delta^{2} \nabla_{h} \nabla_{i} \nabla_{j} \cdots \nabla_{m}+W^{j \cdots m} \Delta^{3} \nabla_{j} \cdots \nabla_{m} \\
+ & 4\left(\nabla^{(a} V^{h i j \cdots m}\right) \Delta \nabla_{a} \nabla_{h} \nabla_{i} \nabla_{j} \cdots \nabla_{m}+4\left(\nabla^{(a} W^{j \cdots m}\right) \Delta^{2} \nabla_{a} \nabla_{j} \cdots \nabla_{m} \\
+ & Y^{i j \cdots m} \Delta^{2} \nabla_{i} \nabla_{j} \cdots \nabla_{m} \\
& + \text { lower order terms. }
\end{aligned}
$$

Moving the Laplacian to the right hand side of each of these terms gives

$$
\Delta^{2} \mathcal{D}=\mathcal{P} \Delta^{2}+4\left(\nabla^{(a} V^{h i j \cdots m)}\right) \nabla_{a} \nabla_{h} \nabla_{i} \nabla_{j} \cdots \nabla_{m} \Delta+\text { lower order terms }
$$

for some differential operator $\mathcal{P}$ and for this to be of the form $\delta \Delta^{2}$ for some differential operator $\delta$ forces (11) to hold, as required.

To find a constraint on $W^{j i k \cdots m}$ we should consider lower order terms:-

$$
\begin{aligned}
\Delta^{2} \mathcal{D}=\mathcal{Q} \Delta^{2}+ & 4\left(\nabla^{(a} V^{h i j k l \cdots m)}\right) \nabla_{a} \nabla_{h} \nabla_{i} \nabla_{j} \nabla_{k} \nabla_{l} \cdots \nabla_{m} \Delta \\
+ & 2\left(\Delta V^{h i j k l \cdots m}\right) \nabla_{h} \nabla_{i} \nabla_{j} \nabla_{k} \nabla_{l} \cdots \nabla_{m} \Delta \\
+ & 4\left(\nabla^{(a} \nabla^{b} V^{h i j k l \cdots m)}\right) \nabla_{a} \nabla_{b} \nabla_{h} \nabla_{i} \nabla_{j} \nabla_{k} \nabla_{l} \cdots \nabla_{m} \\
& +4\left(\nabla^{(a} \nabla^{b} W^{j k l \cdots m)}\right) \nabla_{a} \nabla_{b} \nabla_{j} \nabla_{k} \nabla_{l} \cdots \nabla_{m} \Delta \\
& +4\left(\nabla^{(a} Y^{i j k l \cdots m)}\right) \nabla_{a} \nabla_{i} \nabla_{j} \nabla_{k} \nabla_{l} \cdots \nabla_{m} \Delta \\
& + \text { lower order terms. }
\end{aligned}
$$




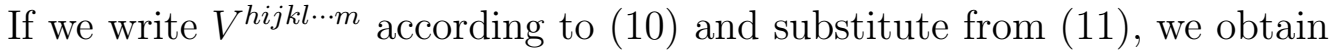

$$
\begin{aligned}
\Delta^{2} \mathcal{D}=\mathcal{R} \Delta^{2}-2(n+2 s-4)\left(\nabla^{(h} \phi^{i j k l \cdots m)}\right) \nabla_{h} \nabla_{i} \nabla_{j} \nabla_{k} \nabla_{l} \cdots \nabla_{m} \Delta \\
+4\left(\nabla^{(h} \phi^{i j k l \cdots m)}\right) \nabla_{h} \nabla_{i} \nabla_{j} \nabla_{k} \nabla_{l} \cdots \nabla_{m} \Delta \\
+4\left(\nabla^{(h} \nabla^{i} W^{j k l \cdots m)}\right) \nabla_{h} \nabla_{i} \nabla_{j} \nabla_{k} \nabla_{l} \cdots \nabla_{m} \Delta \\
\quad+4\left(\nabla^{(h} Y^{i j k l \cdots m)}\right) \nabla_{h} \nabla_{i} \nabla_{j} \nabla_{k} \nabla_{l} \cdots \nabla_{m} \Delta \\
\quad+\text { lower order terms }
\end{aligned}
$$

from which we deduce that

$$
2\left(\nabla^{(h} \nabla^{i} W^{j k l \cdots m)}\right)+2\left(\nabla^{(h} Y^{i j k l \cdots m)}\right)=(n+2 s-6)\left(\nabla^{(h} \phi^{i j k l \cdots m)}\right) .
$$

Passing to the next order, we find

$$
\begin{aligned}
\Delta^{2} \mathcal{D}=\mathcal{R} \Delta^{2}+ & 4\left(\Delta \phi^{a j k l \cdots m)}\right) \nabla_{a} \nabla_{j} \nabla_{k} \nabla_{l} \cdots \nabla_{m} \Delta \\
+ & 4\left(\Delta \nabla^{(a} W^{j k l \cdots m)}\right) \nabla_{a} \nabla_{j} \nabla_{k} \nabla_{l} \cdots \nabla_{m} \Delta \\
+ & 2\left(\Delta Y^{i j k l \cdots m}\right) \nabla_{i} \nabla_{j} \nabla_{k} \nabla_{l} \cdots \nabla_{m} \Delta \\
& +4\left(\nabla^{(a} \nabla^{h} Y^{i j k l \cdots m)}\right) \nabla_{a} \nabla_{h} \nabla_{i} \nabla_{j} \nabla_{k} \nabla_{l} \cdots \nabla_{m} \\
& +4\left(\nabla^{(a} Z^{j k l \cdots m)}\right) \nabla_{a} \nabla_{j} \nabla_{k} \nabla_{l} \cdots \nabla_{m} \Delta \\
& + \text { lower order terms }
\end{aligned}
$$

from which we deduce that

the trace-free part of $\nabla^{(a} \nabla^{h} Y^{i j k l \cdots m)}=0$.

Together with Lemma 1, it follows from (13) that

$$
\text { the trace-free part of } \nabla^{(a} \nabla^{h} \nabla^{i} W^{j k l \cdots m)}=0 \text {, }
$$

as required. This completes the proof of Theorem 1.

In principle, computations such as these are all that is needed to find $\mathcal{D}_{V}$ and $\mathcal{D}_{W}$ as in Theorem 2, For example, we may arrange that (13) holds by taking

$$
Y^{i j k l \cdots m}=\frac{n+2 s-6}{2} \phi^{i j k l \cdots m}
$$

and from (12) we see that $\mathcal{D}_{V}$ must take the form

$$
\mathcal{D}_{V} f=V^{a b \cdots c} \nabla_{a} \nabla_{b} \cdots \nabla_{c} f+\frac{s(n+2 s-6)}{2(n+2 s-2)}\left(\nabla_{a} V^{a b \cdots c}\right) \nabla_{b} \cdots \nabla_{c} f+\cdots
$$

This direct approach, however, is difficult. Fortunately, there is a much easier way of constructing the operators $\mathcal{D}_{V}$ and $\mathcal{D}_{W}$ and this is done in the next section.

\section{The ambient metric and the proof of Theorem 2}

The constructions in this section closely follow those of [6] and so we shall be brief. Let us consider the Lorentzian quadratic form

$$
\tilde{g}_{A B} x^{A} x^{B}=2 x^{0} x^{\infty}+g_{a b} x^{a} x^{b}=\left(x^{0}, x^{a}, x^{\infty}\right)\left(\begin{array}{ccc}
0 & 0 & 1 \\
0 & g_{a b} & 0 \\
1 & 0 & 0
\end{array}\right)\left(\begin{array}{c}
x^{0} \\
x^{b} \\
x^{\infty}
\end{array}\right)
$$


on $\mathbb{R}^{n+2}$. If we embed $\mathbb{R}^{n} \hookrightarrow \mathbb{R P}_{n+1}$ according to

$$
x^{a} \mapsto\left[\begin{array}{c}
1 \\
x^{a} \\
-x^{a} x_{a} / 2
\end{array}\right]
$$

then the action of $\mathrm{SO}(n+1,1)$ on $\mathbb{R}^{n+2}$ preserves $\mathcal{N}$ the null cone of $\tilde{g}_{A B}$ and the corresponding infinitesimal action of $\mathfrak{g}=\mathfrak{s o}(n+1,1)$ on the space of null directions gives rise to conformal Killing vectors on $\mathbb{R}^{n}$. Explicitly, if $\mathfrak{g}$ is realised as skew tensors $V^{B Q}$ on $\mathbb{R}^{n+2}$ in the usual way, then one may check that

$$
V^{B Q} \mapsto V^{b}=\Phi_{B} V^{B Q} \Psi_{Q}^{b}
$$

where

$$
\Phi_{B}=\left(-x^{b} x_{b} / 2, x_{b}, 1\right), \quad \Psi_{Q}^{b}=\left(-x^{b}, \delta^{b}{ }_{q}, 0\right), \quad \delta_{q}^{b}=\text { Kronecker delta. }
$$

In other words,

$$
V^{B Q}=\left(\begin{array}{ccc}
V^{00} & V^{0 q} & V^{0 \infty} \\
V^{b 0} & V^{b q} & V^{b \infty} \\
V^{\infty 0} & V^{\infty q} & V^{\infty \infty}
\end{array}\right)=\left(\begin{array}{ccc}
0 & r^{q} & \lambda \\
-r^{q} & m^{b q} & s^{b} \\
-\lambda & -s^{q} & 0
\end{array}\right)
$$

corresponds to the conformal Killing vector

$$
V^{b}=-s^{b}-m_{q}^{b} x^{q}+\lambda x^{b}+r_{q} x^{q} x^{b}-(1 / 2) x_{q} x^{q} r^{b} .
$$

As in [6], the formula (15) generalises

$$
V^{B Q \cdots C R D S E T} \mapsto V^{b \cdots c d e}=\Phi_{B} \cdots \Phi_{C} \Phi_{D} \Phi_{E} V^{B Q \cdots C R D S E T} \Psi_{Q}^{b} \cdots \Psi_{R}^{c} \Psi_{S}^{d} \Psi_{T}^{e}
$$

to provide an explicit realisation of the isomorphism (5). Here, $V^{B Q \cdots C R D S E T}$ is skew in each pair of indices $B Q, \ldots, C R, D S, E T$, is totally trace-free, and is such that skewing over any three indices gives zero. Similarly, we may take

$$
W^{B Q \cdots C R D E} \in \begin{array}{|l|l|l|l|l|l|l}
\hline & & & & \cdots & & \\
\hline & & & & \cdots & \cdots &
\end{array}
$$

as totally trace-free, skew in each pair $B Q, \ldots, C R$, symmetric in $D E$, and such that skewing over any three indices gives zero. Then (66) is realised by

$$
W^{B Q \cdots C R D E} \mapsto W^{b \cdots c}=\Phi_{B} \cdots \Phi_{C} \Phi_{D} \Phi_{E} W^{B Q \cdots C R D E} \Psi_{Q}^{b} \cdots \Psi_{R}^{c} .
$$

Following Fefferman and Graham [8, we shall use the term 'ambient' to refer to objects defined on $\mathbb{R}^{n+2}$. For example, there is the ambient wave operator

$$
\tilde{\Delta}=\tilde{g}^{A B} \frac{\partial^{2}}{\partial x^{A} \partial x^{B}}
$$

where $\tilde{g}^{A B}$ is the inverse of $\tilde{g}_{A B}$. Let $r=\tilde{g}_{A B} x^{A} x^{B}$ so that $\mathcal{N}=\{r=0\}$. Suppose that $g$ is an ambient function homogeneous of degree $w-2$. A simple calculation gives

$$
\tilde{\Delta}(r g)=r \tilde{\Delta} g+2(n+2 w-2) g .
$$

In particular, if $w=1-n / 2$, then $\tilde{\Delta}(r g)=r \tilde{\Delta} g$. Therefore, if $f$ is homogeneous of degree $1-n / 2$, then $\left.\tilde{\Delta} f\right|_{\mathcal{N}}$ depends only on $\left.f\right|_{\mathcal{N}}$ (since $r g$ provides the freedom 
in extending such a function off $\mathcal{N}$ ). This defines a differential operator on $\mathbb{R}^{n}$ and, as detailed in [7, one may easily verify that it is the Laplacian. This construction is due to Dirac [4] and the main point is that it is manifestly invariant under the action of $\mathfrak{s o}(n+1,1)$. We say that $\Delta$ is conformally invariant acting on conformal densities of weight $1-n / 2$ on $\mathbb{R}^{n}$. This ambient construction of the Laplacian is a simple example of the 'AdS/CFT correspondence' in physics. A principal feature of this correspondence is that calculations are simplified by doing them ambiently or 'in the bulk'. This feature pervades all that follows.

Invariance may also be viewed as follows. Recall that $\mathfrak{g}=\mathfrak{s o}(n+1,1)$ is realised as skew tensors $V^{B Q}$. Each gives rise to an ambient differential operator

$$
\mathfrak{D}_{V}=V^{B Q} x_{B} \frac{\partial}{\partial x^{Q}} \quad \text { where } x_{B}=x^{A} \tilde{g}_{A B} .
$$

It is easily verified that, for $g$ and $f$ of any homogeneity,

$$
\mathfrak{D}_{V}(r g)=r \mathfrak{D}_{V} g \text { and } \tilde{\Delta} \mathfrak{D}_{V} f=\mathfrak{D}_{V} \tilde{\Delta} f .
$$

The first of these implies that $\mathfrak{D}_{V}$ induces differential operators on $\mathbb{R}^{n}$ for densities of any conformal weight: simply extend the corresponding homogeneous function on $\mathcal{N}$ into $\mathbb{R}^{n+2}$, apply $\mathfrak{D}_{V}$, and restrict back to $\mathcal{N}$. In particular, let us denote by $\mathcal{D}_{V}$ and $\delta_{V}$ the differential operators so induced on densities of weight $1-n / 2$ and $-1-n / 2$, respectively. Bearing in mind the ambient construction of the Laplacian, it follows immediately from the second equation of (23) that $\Delta \mathcal{D}_{V}=\delta_{V} \Delta$. In other words, the infinitesimal conformal invariance of $\Delta$ gives rise to the symmetries $\mathcal{D}_{V}$ as differential operators.

The formula (22) generalises to provide further symmetries. It is shown in 6 that the ambient differential operator

$$
\mathfrak{D}_{V}=V^{B Q \cdots C R D S E T} x_{B} \cdots x_{C} x_{D} x_{E} \frac{\partial^{s}}{\partial x^{Q} \cdots \partial x^{R} \partial x^{S} \partial x^{T}}
$$

provides a symmetry of the Laplacian for all

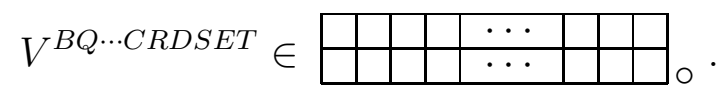

The proof of Theorem 2 for conformal Killing tensors is essentially contained in the following:-

Proposition 1. The ambient operator (24) induces a symmetry of $\Delta^{2}$. The symbol of this operator is given by (18).

Proof. Firstly, we need to know the ambient description of $\Delta^{2}$. Iterating (21) gives

$$
\tilde{\Delta}^{2}(r g)=r \tilde{\Delta}^{2} g+4(n+2 w-4) \tilde{\Delta} g .
$$

In particular, if $w=2-n / 2$, then $\tilde{\Delta}^{2}(r g)=r \tilde{\Delta}^{2} g$. Therefore, if $f$ is homogeneous of degree $2-n / 2$, then $\left.\tilde{\Delta}^{2} f\right|_{\mathcal{N}}$ depends only on $\left.f\right|_{\mathcal{N}}$ and it is shown in [7] that the resulting differential operator on $\mathbb{R}^{n}$ is $\Delta^{2}$. It is it easily verified that (23) holds more generally for $\mathfrak{D}_{V}$ of the form (24). It follows that

$$
\mathfrak{D}_{V}(r g)=r \mathfrak{D}_{V} g \quad \text { and } \quad \tilde{\Delta}^{2} \mathfrak{D}_{V} f=\mathfrak{D}_{V} \tilde{\Delta}^{2} f
$$


for $f$ and $g$ of any homogeneity. Arguing as for the Laplacian shows that the operator $\mathcal{D}_{V}$ on $\mathbb{R}^{n}$ obtained from $\mathfrak{D}_{V}$ acting on functions homogeneous of degree $w=2-n / 2$ is a symmetry of $\Delta^{2}$. There are some details to be verified to make sure that the symbol of $\mathcal{D}_{V}$ is given by (18). However, similar verifications are done in [6] and we leave them to the interested reader.

The ambient construction of symmetries from tensors $W^{B Q \cdots C R D E}$ as in (19) is less obvious. The following proposition completes the proof of Theorem 2.

Proposition 2. For any tensor $W^{B Q \cdots C R D E}$ satisfying the symmetries of (19) the ambient differential operator

$$
W^{B Q \cdots C R D E} x_{B} \cdots x_{C}\left(x_{D} x_{E} \tilde{\Delta}-2 x_{D} \frac{\partial}{\partial x^{E}}\right) \frac{\partial^{s-2}}{\partial x^{Q} \cdots \partial x^{R}}
$$

induces a symmetry $\mathcal{D}_{W}$ of $\Delta^{2}$ of the form

$$
\mathcal{D}_{W}=W^{b \ldots c} \Delta \nabla_{b} \cdots \nabla_{c}+\text { lower order terms }
$$

where $W^{b \cdots c}$ is given by (20).

It is not too hard to prove this Proposition by direct calculation along the lines of Proposition 1. There is a difference, however, in that the analogue of the first equation of (25) holds only for $g$ of homogeneity $-n / 2$. Moreover, for an analogue of the second equation, one needs to use the ambient operator

$$
W^{B Q \cdots C R D E} x_{B} \cdots x_{C}\left(x_{D} x_{E} \tilde{\Delta}+6 x_{D} \frac{\partial}{\partial x^{E}}\right) \frac{\partial^{s-2}}{\partial x^{Q} \cdots \partial x^{R}}
$$

on the right hand side and, even so, it is valid only for homogeneity $2-n / 2$. Of course, these homogeneities are exactly what we need for $\Delta^{2}$ but there is a more satisfactory ambient construction giving rise to exactly the same symmetries, which we shall defer to the following section. The operators in this more satisfactory construction enjoy the proper generalisation of (25), namely (29).

\section{The Proof of Theorem 3}

We shall prove Theorem 3 by a new method, improving on [6]. As a side effect, we shall obtain a straightforward proof of Proposition 2.

In the previous section, we found in (22) a linear mapping

$$
\mathfrak{s o}(n+1,1)=\mathfrak{g} \ni V \longmapsto \mathfrak{D}_{V},
$$

where $\mathfrak{D}_{V}$ is an ambient differential operator acting on functions homogeneous of degree $w$ for any $w$. By dint of (23), we obtain an induced linear mapping

$$
\mathfrak{g} \ni V \longmapsto \mathcal{D}_{V}
$$

where $\mathcal{D}_{V}$ is a differential operator acting on conformal densities of weight $w$. In fact, it is shown in [6] that

$$
\mathcal{D}_{V} f=V^{a} \nabla_{a} f-\frac{w}{n}\left(\nabla_{a} V^{a}\right) f
$$

where $V^{a}$ is the conformal Killing vector associated to $V \in \mathfrak{g}$ according to (15). 
The mapping (26) immediately extends to the whole tensor algebra $\otimes \mathfrak{g}$ by

$$
\mathfrak{g} \otimes \mathfrak{g} \otimes \cdots \otimes \mathfrak{g} \ni U \otimes \cdots \otimes V=X \mapsto \mathfrak{D}_{X} \equiv \mathfrak{D}_{U} \cdots \mathfrak{D}_{V}
$$

and extended by linearity. It follows from (23) that

$$
\mathfrak{D}_{X}(r g)=r \mathfrak{D}_{X} g \text { and } \tilde{\Delta} \mathfrak{D}_{X}=\mathfrak{D}_{X} \tilde{\Delta}
$$

and hence that there is an induced series of operators $\mathcal{D}_{X}$ acting on densities of weight $w$ on $\mathbb{R}^{n}$ and providing symmetries of $\Delta^{k}$ when $w=k-n / 2$. Of course, for simple tensors $X$ these operators are obtained by composing the basic operators $\mathcal{D}_{V}$ for $V \in \mathfrak{g}$. However, usefully to compute even the basic composition $\mathcal{D}_{U} \mathcal{D}_{V}$ from (27) for $U, V \in \mathfrak{g}$ is difficult. Though this is done in [6], the simpler approach adopted there is to compute $\mathfrak{D}_{U} \mathfrak{D}_{V}$ instead. The object is to see how this composition breaks up under (77) but, for this purpose, the following argument is even more straightforward.

We compute

$$
\mathfrak{D}_{U} \mathfrak{D}_{V}=U^{B Q} x_{B} \frac{\partial}{\partial x^{Q}} V^{C R} x_{C} \frac{\partial}{\partial x^{R}}=U^{B Q} V^{C R} x_{B} x_{C} \frac{\partial^{2}}{\partial x^{Q} \partial x^{R}}+U^{B Q} V_{Q}^{R} x_{B} \frac{\partial}{\partial x^{R}},
$$

which extends by linearity to give

$$
\mathfrak{D}_{X}=X^{B Q C R} x_{B} x_{C} \frac{\partial^{2}}{\partial x^{Q} \partial x^{R}}+X^{B Q}{ }_{Q}^{R} x_{B} \frac{\partial}{\partial x^{R}}, \quad \text { for } X^{B Q C R} \in \mathfrak{g} \otimes \mathfrak{g} .
$$

We can simply apply this formula to tensors $X$ from each of the summands on the right hand side of (17). All $X^{B Q C R}$ in $\mathfrak{g} \otimes \mathfrak{g}$ are skew in $B Q$ and $C R$ but the various summands of (7) are characterised as follows.

$$
\square_{0} \longleftrightarrow\left\{\begin{array}{l}
X^{B Q C R}+X^{B C R Q}+X^{B R Q C}=0 \\
X^{B Q C R} \text { is totally trace-free. }
\end{array}\right.
$$

Therefore, the second term in (30) vanishes and $\mathfrak{D}_{X}$ is given by (24), as expected.

Next we have

$$
\square \circ \leftrightarrow X^{B Q C R}=W^{B C} \tilde{g}^{Q R}-W^{Q C} \tilde{g}^{B R}-W^{B R} \tilde{g}^{Q C}+W^{Q R} \tilde{g}^{B C}
$$

where $W^{B C}$ is symmetric trace-free. Therefore,

$$
\mathfrak{D}_{X}=W^{B C} x_{B} x_{C} \tilde{\Delta}-2 W^{Q C} x_{C} x^{R} \frac{\partial^{2}}{\partial x^{R} \partial x^{Q}}-n W^{B R} x_{B} \frac{\partial}{\partial x^{R}}+W^{Q R} r \frac{\partial^{2}}{\partial x^{Q} \partial x^{R}}
$$

and, when acting on functions homogeneous of degree $w$,

$$
\mathfrak{D}_{X}=W^{B C}\left(x_{B} x_{C} \tilde{\Delta}-(n+2 w-2) x_{B} \frac{\partial}{\partial x^{C}}+r \frac{\partial^{2}}{\partial x^{B} \partial x^{C}}\right) .
$$

There are two immediate consequences of this formula. Firstly, when $w=1-n / 2$, the appropriate homogeneity for the Laplacian, we obtain

$$
\mathfrak{D}_{X}=W^{B C}\left(x_{B} x_{C} \tilde{\Delta}+r \frac{\partial^{2}}{\partial x^{B} \partial x^{C}}\right)
$$


and the induced operator $\mathcal{D}_{X}$ on $\mathbb{R}^{n}$ is clearly of the form $\mathcal{P} \Delta$. Therefore, this summand in the decomposition (17) is contained in the annihilator ideal. This is confirmed by (8) . On the other hand, when $w=2-n / 2$, we obtain

$$
\mathfrak{D}_{X}=W^{B C}\left(x_{B} x_{C} \tilde{\Delta}-2 x_{B} \frac{\partial}{\partial x^{C}}+r \frac{\partial^{2}}{\partial x^{B} \partial x^{C}}\right)
$$

and the induced operator on $\mathbb{R}^{n}$ coincides with the statement of Proposition 2 in this case. It is also easy to compute the symbol of the induced operator on $\mathbb{R}^{n}$ as follows.

\section{Lemma 2.}

$$
\tilde{g}^{Q R} \Psi_{Q}^{b} \Psi_{R}^{c}=g^{b c} \quad \Phi_{B} \tilde{g}^{B Q} \Psi_{Q}^{b}=0 \quad \Phi_{B} \Phi_{C} \tilde{g}^{B C}=0
$$

Proof. These are simple computations from (16).

From this lemma, if $X^{B Q C R}$ is of the form given in (31), then

$$
\Phi_{B} \Phi_{C} X^{B Q C R} \Psi_{Q}^{b} \Psi_{R}^{c}=\Phi_{B} \Phi_{C} W^{B C} g^{b c}=W g^{b c}
$$

where $W$ is given by (20). It follows that the induced operator on $\mathbb{R}^{n}$ is of the form

$$
W g^{a b} \nabla_{a} \nabla_{b}+\text { lower order terms }=W \Delta+\text { lower order terms. }
$$

Therefore, we have proved Proposition 2 for second order operators. Let us return to analysing the effect of the various summands of $\mathfrak{g} \otimes \mathfrak{g}$ in (30).

Next we have

$$
\mathbb{R} \leftrightarrow X^{B Q C R}=V \frac{1}{n(n+1)(n+2)}\left(\tilde{g}^{Q C} \tilde{g}^{B R}-\tilde{g}^{B C} \tilde{g}^{Q R}\right) \quad \text { for constant } V .
$$

The normalisation is arranged so that the Killing form $\langle\rangle:, \mathfrak{g} \otimes \mathfrak{g} \rightarrow \mathbb{R}$ gives

$$
\begin{aligned}
X^{B Q C R} \longmapsto-n X^{B Q}{ }_{B Q} & =-n V \frac{1}{n(n+1)(n+2)}\left(\delta^{Q}{ }_{B} \delta^{B}{ }_{Q}-\delta^{B}{ }_{B} \delta^{Q}{ }_{Q}\right) \\
& =-n V \frac{1}{n(n+1)(n+2)}\left((n+2)-(n+2)^{2}\right)=V .
\end{aligned}
$$

We compute

$$
\mathfrak{D}_{X}=V \frac{1}{n(n+1)(n+2)}\left(x^{Q} x^{R} \frac{\partial^{2}}{\partial x^{R} \partial x^{Q}}+r \tilde{\Delta}+(n+1) x^{R} \frac{\partial}{\partial x^{R}}\right) .
$$

Therefore, when acting on functions homogeneous of degree $w$,

$$
\mathfrak{D}_{X}=V \frac{1}{n(n+1)(n+2)}(r \tilde{\Delta}+w(n+w)) \text {. }
$$

Hence, the corresponding action of $\mathcal{D}_{X}$ on $\mathbb{R}^{n}$ is

$$
\mathcal{D}_{X} f=\frac{w(n+w)}{n(n+1)(n+2)} V f
$$

for conformal densities of weight $w$. In particular, if $w=1-n / 2$ then

$$
\mathcal{D}_{X} f=-\frac{(n-2)}{4 n(n+1)} V f .
$$

This is exactly as predicted in (8). If $w=2-n / 2$, however, then

$$
\mathcal{D}_{X} f=-\frac{(n-4)(n+4)}{4 n(n+1)(n+2)} V f
$$

in agreement with (9). 
Next we have

$$
\square_{0} \longleftrightarrow\left\{\begin{array}{l}
X^{B Q C R}+X^{C R B Q}=0 \\
X^{B Q C R} \text { is totally trace-free }
\end{array}\right.
$$

In this case both terms in (30) evidently vanish.

Next we have

$$
\square \leftrightarrow X^{B Q C R}=\frac{1}{2 n}\left(V^{B R} \tilde{g}^{Q C}-V^{Q R} \tilde{g}^{B C}-V^{B C} \tilde{g}^{Q R}+V^{Q C} \tilde{g}^{B R}\right)
$$

where $V^{B R}$ is skew. The normalisation is arranged so that the Lie bracket $\mathfrak{g} \otimes \mathfrak{g} \rightarrow \mathfrak{g}$ gives

$$
X^{B Q C R} \longmapsto X^{B}{ }_{Q}^{Q R}-X^{R}{ }_{Q}^{Q B}=\frac{1}{2} V^{B R}-\frac{1}{2} V^{R B}=V^{B R} .
$$

Since $X^{B Q C R}=-X^{C R B Q}$, the first term in (30) vanishes. Therefore,

$$
\mathfrak{D}_{X}=X^{B Q}{ }_{Q}^{R} x_{B} \frac{\partial}{\partial x^{R}}=\frac{1}{2} V^{B R} x_{B} \frac{\partial}{\partial x^{R}}=\frac{1}{2} \mathfrak{D}_{V} .
$$

This accounts for the term $\frac{1}{2} \mathfrak{D}_{[V, W]}$ in both (8) and (9).

The final summand corresponds to totally skew tensors $X^{B Q C R}$ and for these it is clear that $\mathfrak{D}_{X}$ given by (30) vanishes. It accounts for the presence of this summand in the ideals defined by (8) or (9).

In summary, by considering the effect in (30) of tensors from the various summands in the decomposition (7) of $\mathfrak{g} \otimes \mathfrak{g}$, we have verified (8) and (9). It is also worthwhile recording what we have shown for a general conformal weight $w$.

Theorem 4. Suppose that $X^{a}$ and $Y^{a}$ are conformal Killing vector fields on $\mathbb{R}^{n}$ corresponding to $X^{A P}$ and $Y^{A P}$, respectively, in $\mathfrak{g}=\mathfrak{s o}(n+1,1)$. Then

$$
\mathcal{D}_{X} \mathcal{D}_{Y} f=\mathcal{D}_{X \odot Y} f+\mathcal{D}_{X \bullet Y} f+\frac{1}{2} \mathcal{D}_{[X, Y]} f+\frac{w(n+w)}{n(n+1)(n+2)}\langle X, Y\rangle f
$$

on densities of weight $w$. Here,

* $\mathcal{D}_{X} f=X^{a} \nabla_{a} f-\frac{w}{n}\left(\nabla_{a} X^{a}\right) f$.

$*(X \odot Y)^{a b}=\frac{1}{2} X^{a} Y^{b}+\frac{1}{2} X^{b} Y^{a}-\frac{1}{n} X^{c} Y_{c} g^{a b}$ is a conformal Killing tensor and for a general conformal Killing tensor $V^{a b}$,

$$
\mathcal{D}_{V} f=V^{a b} \nabla_{a} \nabla_{b} f-\frac{2(w-1)}{n+2}\left(\nabla_{a} V^{a b}\right) \nabla_{b} f+\frac{w(w-1)}{(n+2)(n+1)}\left(\nabla_{a} \nabla_{b} V^{a b}\right) f .
$$

$* X \bullet Y=\frac{1}{n} X^{a} Y_{a}=W$ satisfies $\nabla_{a} \nabla_{b} \nabla_{c} W=g_{(a b} \phi_{c)}$ and, for such a field in general,

$$
\mathcal{D}_{W} f=W \Delta f-\frac{n+2 w-2}{2}\left(\nabla^{a} W\right) \nabla_{a} f+\frac{w(n+2 w-2)}{2(n+2)}(\Delta W) f .
$$

$*[X, Y]^{a}=X^{b} \nabla_{b} Y^{a}-Y^{b} \nabla_{b} X^{a}$ is a conformal Killing field.

$*\langle X, Y\rangle=\left(\nabla_{b} X^{a}\right)\left(\nabla_{a} Y^{b}\right)-\frac{n-2}{n^{2}}\left(\nabla_{a} X^{a}\right)\left(\nabla_{b} Y^{b}\right)-\frac{2}{n} X^{a} \nabla_{a} \nabla_{b} Y^{b}-\frac{2}{n} Y^{a} \nabla_{a} \nabla_{b} X^{b}$ is constant.

Within $(\mathfrak{g} \otimes \mathfrak{g}) \oplus \mathfrak{g} \oplus \mathbb{R}$, however, these operations are defined as in Section 2 . 
Proof. Apart from $W=X \bullet Y$ the various formulae have just been established or are taken from [6] (with a minor rearrangement for $\langle X, Y\rangle$ on $\mathbb{R}^{n}$ ). To complete the proof, therefore, it remains to establish the formula on $\mathbb{R}^{n}$ for $X \bullet Y$ and the formula for $\mathcal{D}_{W}$ in general. One possibility is to compute the composition $\mathcal{D}_{X} \mathcal{D}_{Y}$ in full and collect terms. Though this certainly works, there is a short cut as follows. Certainly,

$$
\begin{aligned}
\mathcal{D}_{X} \mathcal{D}_{Y} & =X^{a} Y^{b} \nabla_{a} \nabla_{b}+\text { lower order terms } \\
& =\left(\frac{1}{2} X^{a} Y^{b}+\frac{1}{2} X^{b} Y^{a}-\frac{1}{n} X^{c} Y_{c} g^{a b}\right) \nabla_{a} \nabla_{b}+\frac{1}{n} X^{c} Y_{c} \Delta+\text { lower order terms } \\
& =(X \odot Y)^{a b} \nabla_{a} \nabla_{b}+\frac{1}{n} X^{c} Y_{c} \Delta+\text { lower order terms. }
\end{aligned}
$$

It follows that $X \bullet Y=\frac{1}{n} X^{a} Y_{b}$, as advertised. Rather than find the lower order terms by direct computation, we claim that they are forced by invariance under the conformal action of $\mathfrak{s o}(n+1,1)$. This is essentially the argument used in [6, §5] to find explicit formulae for $\mathcal{D}_{V}$ in case of an arbitrary conformal Killing tensor $V^{b c \cdots d}$ acting on densities of any weight. In our case, the argument is as follows. We are looking for a differential operator of the form

$$
\mathcal{D}_{W} f=W \Delta f+\alpha\left(\nabla^{a} W\right) \nabla_{a} f+\beta(\Delta W) f
$$

and it remains to determine the constants $\alpha$ and $\beta$ in order that such an operator be conformally invariant under flat-to-flat rescalings of the standard metric on $\mathbb{R}^{n}$. We shall follow the conventions of [1] concerning conformal geometry. If $\hat{g}_{a b}=\Omega^{2} g_{a b}$ is also a flat metric, then

$$
\nabla^{a} \Upsilon_{a}=-\frac{n-2}{2} \Upsilon^{a} \Upsilon_{a} \quad \text { for } \Upsilon_{a}=\left(\nabla_{a} \Omega\right) / \Omega
$$

Now $W$ has conformal weight 2 and we are supposing that $f$ has conformal weight $w$. It follows that

$$
\begin{aligned}
\hat{\nabla}_{a} f & =\nabla_{a} f+w \Upsilon_{a} f \\
\hat{\nabla}^{a} W & =\nabla^{a} W+2 \Upsilon^{a} W \\
\hat{\Delta} f & =\Delta f+(n+2 w-2)\left(\Upsilon^{a} \nabla_{a} f+\frac{w}{2} \Upsilon^{a} \Upsilon_{a} f\right) \\
\hat{\Delta} W & =\Delta W+(n+2)\left(\Upsilon^{a} \nabla_{a} W+\Upsilon^{a} \Upsilon_{a} W\right)
\end{aligned}
$$

whence (32) satisfies $\hat{\mathcal{D}}_{W}=\mathcal{D}_{W}$ if and only if

$$
\alpha=-\frac{n+2 w-2}{2} \text { and } \beta=\frac{w(n+2 w-2)}{2(n+2)},
$$

as required.

Notice that (2) and (3) are special cases of Theorem 4. Also notice that $\mathcal{D}_{W}=W \Delta$ when $w=1 / n-2$, which explains the absence of $X \bullet Y$ in the generators (8) of the annihilator ideal in this case.

We shall now complete the proof of Proposition 2 (and, hence, of Theorem 2).

Lemma 3. Suppose that

$$
X^{B Q \cdots C R D S E T} \in \square \odot \cdots \odot \square \odot \square \odot \square=\odot^{s} \mathfrak{g} \subset \bigotimes^{s} \mathfrak{g} .
$$


In other words, $X^{B Q \cdots C R D S E T}$ is skew in each pair of indices $B Q, \ldots, C R, D S, E T$, has $2 s$ indices in total, and is invariant under permutations of the paired indices. Then the operator defined by (28) is

$$
\begin{aligned}
\mathfrak{D}_{X}=X^{B Q \cdots C R D S E T} x_{B} \cdots x_{C} x_{D} x_{E} \frac{\partial^{s}}{\partial x^{Q} \cdots \partial x^{R} \partial x^{S} \partial x^{T}} \\
+ \\
+\frac{s(s-1)}{2} X^{B Q \cdots C R D S}{ }_{S}^{T} x_{B} \cdots x_{C} x_{D} \frac{\partial^{s-1}}{\partial x^{Q} \cdots \partial x^{R} \partial x^{T}} \\
+ \text { lower order terms. }
\end{aligned}
$$

Proof. The derivation of (30) from (22) is easily extended by induction.

Suppose that $W^{B Q \cdots C R D E}$ satisfies the symmetries of (19) as in the statement of Proposition 2. Generalising (31), let $X^{B Q \cdots C R D S E T}$ be obtained by forming

$$
W^{B Q \cdots C R D E} \tilde{g}^{S T}-W^{B Q \cdots C R S E} \tilde{g}^{D T}-W^{B Q \cdots C R S E} \tilde{g}^{D T}+W^{B Q \cdots C R S T} \tilde{g}^{D E}
$$

and then symmetrising over the paired indices $B Q, \ldots, C R, D S, E T$. From Lemma 3 , a short calculation gives

$$
\begin{gathered}
\mathfrak{D}_{X}=W^{B Q \cdots C R D E} x_{B} \cdots x_{C}\left(x_{D} x_{E} \tilde{\Delta}-2 x_{D} x^{S} \frac{\partial^{2}}{\partial x^{S} \partial x^{E}}+r \frac{\partial^{2}}{\partial x^{D} \partial x^{E}}\right) \frac{\partial^{s-2}}{\partial x^{Q} \cdots \partial x^{R}} \\
-(n+2 s-4) W^{B Q \cdots C R D E} x^{B} \cdots x^{C} x^{D} \frac{\partial^{s-1}}{\partial x^{E} \partial x^{Q} \cdots \partial x^{R}}
\end{gathered}
$$

where $W^{B Q \cdots C R D E}$ being trace-free ensures that there are no lower order terms. Therefore, when acting on functions homogeneous of degree $w$, we find

$\mathfrak{D}_{X}=W^{B Q \cdots C R D E} x_{B} \cdots x_{C}\left(x_{D} x_{E} \tilde{\Delta}-(n+2 w-2) x_{D} \frac{\partial}{\partial x^{E}}+r \frac{\partial^{2}}{\partial x^{D} \partial x^{E}}\right) \frac{\partial^{s-2}}{\partial x^{Q} \cdots \partial x^{R}}$ and, in particular, if $w=2-n / 2$ then we have completed the proof of Proposition 2

It remains to finish the proof of Theorem 3, As in [6], this is done by considering the corresponding graded algebra $\operatorname{gr}\left(\mathcal{B}_{n}\right)$. From Corollary 1 we know the structure of this algebra - as a vector space it is (41) and its algebra structure arises from its being a quotient of the tensor algebra $\otimes \mathfrak{g}$. Theorem 4 with $w=2-n / 2$ implies that the elements (9) are contained in the ideal defining $\mathcal{B}_{n}$, namely the kernel of the mapping $\otimes \mathfrak{g} \rightarrow \mathcal{B}_{n}$. From these elements alone, the corresponding graded ideal contains

$$
V \otimes W-V \odot W-V \bullet W \text { for } V, W \in \mathfrak{g} .
$$

Let us consider the ideal generated by these elements alone, i.e. generated by $\mathcal{I}_{2}$ where we have grouped the decomposition (77) according to

$$
\boxminus \otimes \boxminus=\square_{\circ} \oplus \square \text { ๑ } \oplus \mathcal{I}_{2}
$$

In particular, $\mathcal{I}_{2}$ contains $\mathfrak{g} \wedge \mathfrak{g}$ and $\operatorname{so} \operatorname{gr}\left(\mathcal{B}_{n}\right)$ is a quotient of the symmetric tensor algebra $\bigodot^{s} \mathfrak{g}$. We have just seen how the differential operators $\mathcal{D}_{W}$ in Proposition 2 and hence Theorem 2 arise - the representation (19) is realised as a specific submodule of $\bigodot^{s} \mathfrak{g}$ and, indeed, this is the unique submodule of this type. Hence, as a vector space, for $s \geq 2$ we may write $\mathcal{K}_{n, s} \oplus \mathcal{L}_{n, s} \subset \bigodot^{s} \mathfrak{g}$ and the corresponding symmetry 
operators are given by the ambient construction in a uniform fashion (as developed earlier in this section). More specifically,

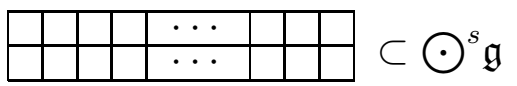

consists of those $X^{B Q \cdots C R D S E T}$ such that skewing over any three indices gives zero and then

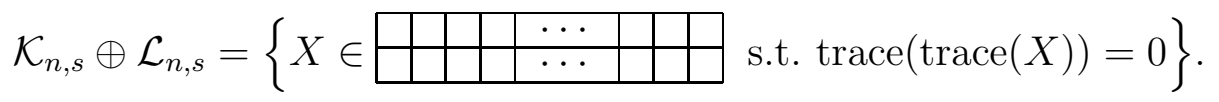

For convenience, let us write $\mathcal{K}_{n, s} \oplus \mathcal{L}_{n, s} \equiv \mathcal{M}_{n, s}$. From this viewpoint it is easy to see that the two-sided ideal generated by $\mathcal{I}_{2}$ in $\otimes \mathfrak{g}$ is not big enough to have (41) as its quotient and the problem is when $s=4$. Arguing as in [6], or more specifically as in [5. Theorem 3], we would like to show that

$$
\mathcal{M}_{n, s}=\left(\mathcal{M}_{n, s-1} \otimes \square\right) \cap\left(\square \otimes \mathcal{M}_{n, s-1}\right) \text { for } s \geq 3
$$

but this is not true when $s=4$ and the problem is with traces. More specifically, it is shown in [5, Theorem 2] and, in any case, is easily verified as in [6] that

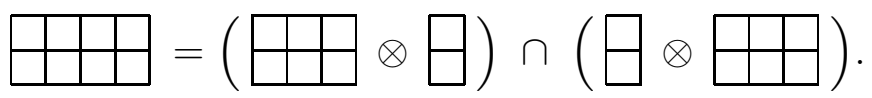

Therefore, we are asking whether a tensor $X^{B Q C R D S E T}$ enjoying the symmetries of the left hand side of (34) and such that

$$
X_{B Q}^{B Q}{ }^{D S E T}=0 \text { and } X_{D S}^{B Q C R D S}=0
$$

has the property that all its second traces vanish. This is not the case. Indeed, a counterexample may be constructed from any trace-free symmetric tensor $Z^{B C D E}$. Let $\tilde{g}^{Q R S T} \equiv \tilde{g}^{(Q R} \tilde{g}^{S T)}$ and then

$$
X^{B Q C R D S E T}=\operatorname{skew}\left(Z^{B C D E} \tilde{g}^{Q R S T}\right)
$$

where 'skew' means to take the skew part in the index pairs $B Q, C R, D S, E T$ (thus generalising (31) to $\square \square \square \square$ o). It is readily verified that (35) are satisfied but that

$$
X_{Q}^{B Q C}{ }_{Q}^{D S E}{ }_{S}=\text { a non-zero multiple of } Z^{B C D E} \text {. }
$$

The proof of Theorem 3 now reduces to the following two facts. The first is that the tensor $X \in \bigodot^{4} \mathfrak{g}$ constructed in (36) induces a non-zero multiple of the differential operator

$$
W^{B C D E} x_{B} x_{C} x_{D} x_{E} \Delta^{2}
$$

on $\mathbb{R}^{n}$, which we decreed to be equivalent to zero in Definition 2. On the one hand this shows that $\square \square \mid \square$ o should be included in the annihilator ideal for $\mathcal{B}_{n}$ as stated in Theorem 3. On the other hand, the second easy fact is that (33) is true for $s \neq 4$ and this implies that no further additions to the ideal are necessary. The first fact is an elementary calculation. Both will be left to the reader. 


\section{REFERENCES}

[1] R.J. Baston and M.G. Eastwood, Invariant operators, Twistors in Mathematics and Physics, Lond. Math. Soc. Lecture Notes vol. 156, Cambridge University Press 1990, pp. 129-163.

[2] C.P. Boyer, E.G. Kalnins, and W. Miller, Jr., Symmetry and separation of variables for the Helmholtz and Laplace equations, Nagoya Math. Jour. 60 (1976) 35-80.

[3] T.P. Branson, A. Čap, M.G. Eastwood, and A.R. Gover, Prolongations of geometric overdetermined systems, Int. Jour. Math. 17 (2006) 641-664.

[4] P.A.M. Dirac, The electron wave equation in de-Sitter space, Ann. Math. 36 (1935) 657-669.

[5] M.G. Eastwood, The Cartan product, Bull. Belg. Math. Soc. 11 (2004) 641-651.

[6] M.G. Eastwood, Higher symmetries of the Laplacian, Ann. Math. 161 (2005) 1645-1665.

[7] M.G. Eastwood and C.R. Graham. Invariants of conformal densities, Duke Math. Jour. 63 (1991) 633-671.

[8] C. Fefferman and C.R. Graham, Conformal invariants, Élie Cartan et les Mathématiques d'Aujourdui, Astérisque 1985, pp. 95-116.

[9] J. Lepowsky, A generalization of the Bernstein-Gelfand-Gelfand resolution, Jour. Alg. 49 (1977), 496-511.

[10] A.G. Nikitin. Generalized Killing tensors of arbitrary rank and order, Ukrain. Mat. Zh. 43 (1991) 786-795.

[11] A.G. Nikitin and A.I. Prilipko, Generalized Killing tensors and the symmetry of the KleinGordon-Fock equation, Akad. Nauk Ukrain. SSR Inst. Mat., preprint, 1990.

School of Mathematical Sciences, University of Adelaide, SA 5005, Australia

E-mail address: meastwoo@member.ams.org

School of Mathematical Sciences, University of Adelaide, SA 5005, Australia

E-mail address: tleistne@maths.adelaide.edu.au 